\title{
Respiratory Volume Monitoring in the Perioperative Setting Across Multiple Centers
}

\author{
Chunyuan Qiu, Eugene Cheng, Shawn R Winnick, Vu T Nguyen, Fang-Chen Hou, \\ Sally Shou Yen, Gonzalo D Custodio, Jennifer H Dang, Diana LaPlace, Atef Morkos, \\ Elena P Chung, and Vimal N Desai
}

BACKGROUND: The prevalence of nuisance (technical) alarms is the leading cause of alarm fatigue resulting in decreased awareness and a reduction in effective care. The Joint Commission identified in their National Patient Safety goals alarm fatigue as a major safety issue. The introduction of noninvasive respiratory volume monitoring (RVM) has implications for effective perioperative respiratory status management. We evaluated this within the Kaiser Permanente health system. METHODS: This observational study was conducted at $\mathbf{4}$ hospitals in the Kaiser Permanente system. Standard data from RVM, pulse oximetry, and capnography were collected postoperatively in the post-anesthesia care unit (PACU) and/or on the general hospital floor. Devicespecific alarm types, rates, and respective actions were recorded and analyzed by non-study staff. RESULTS: RVM was applied to 247 subjects ( 143 females, body mass index $32.3 \pm 8.7 \mathrm{~kg} / \mathrm{m}^{2}$, age $60.9 \pm 13.9$ y) providing $2,321 \mathrm{~h}$. RVM alarms occurred 605 times $(0.25$ alarms/h); $64 \%$ were actionable and addressed, $17 \%$ were not addressed, $13 \%$ were self-resolved, and only $6 \%$ were nuisance. In a subgroup, RVM completed all $127 \mathrm{~h}$ of monitoring, whereas oximetry with capnography only completed $51 \mathrm{~h}$ with 12.9 alarms/h (73\% nuisance). The overall RVM alarm rate was significantly lower than with either pulse oximeters or capnography monitors. We saw a nearly 1,000-fold reduction in nuisance alarms compared to capnography and a 20-50-fold reduction in nuisance alarms compared to pulse oximetry. CONCLUSIONS: Our study indicates that alarm fatigue due to nuisance alarms continues to be a clinical challenge in perioperative settings. Among the 3 common technologies for respiratory function monitoring, RVM had the lowest rate of overall technical alarms and the highest rate of compliance. Furthermore, with early interventions, none of the subjects monitored with RVM suffered any negative outcomes. Key words: respiratory volume monitoring; noninvasive tidal volume; alarm fatigue; perioperative monitoring; alarms; monitoring; respiratory; anesthesia; PACU. [Respir Care 2020;65(4):482-491. (C) 2020 Daedalus Enterprises]

\section{Introduction}

Numerous physiologic monitors have been introduced into perioperative practice; however, there have been chal-

\footnotetext{
Drs Qiu, LaPlace, Morkos, Chung, Desai, and Nguyen, as well as Ms Hou, Ms Yen, Mr Custodio, and Ms Dang, are affiliated with the Department of Anesthesiology, Kaiser Permanente Baldwin Park Medical Center, Baldwin Park, California. Dr Cheng is affiliated with the Department of Anesthesiology, Kaiser Permanente San Jose Medical Center, San Jose, California. Dr Winnick is affiliated with the Department of Anesthesiology, Kaiser Permanente Fontana Ontario Medical Center, Fontana, California.
}

Drs Qiu and Desai presented a version of this paper at the Military Health System Research Symposium 2018, held August 20-23, 2019, in Kissim- lenges in demonstrating clinical advantage and improvement in patient experience. ${ }^{1}$ While some issues can be attributed to the interface between technology and the physiology of the patient, often the difficulty is in finding a mee, Florida, and at the American Society of Anesthesiologists 2018
Annual Meeting, held October 13-17, 2018, in San Francisco, California.
Ms Dang, Mr Custodio, Ms Trieu, and Mr Depakakibo presented a ver-
sion of this paper at the American Society of PeriAnesthesia Nurses
Annual Conference 2019, held May 5-9, 2019, in Nashville, Tennessee.
The authors have disclosed that Respiratory Motion loaned the monitors
for the evaluation and provided the disposables free of charge. The man-
ufacturer reviewed a draft of the paper and made technical suggestions,
but did not participate in the final iteration or review of the paper. 
balance between reliable and actionable data versus excessive nonactionable nuisance alarms. Not surprisingly, excessive nuisance alarms are the leading cause of alarm fatigue for medical professionals, which decreases effective awareness of a patient's true status and inappropriately diverts the attention of care providers from other patients. False alarms can thereby negate monitoring efficacy and patient safety benefits. ${ }^{2}$ The Joint Commission identified in their National Patient Safety Goals alarm fatigue as a major safety risk $^{3}$; in January of 2016, as part of their phase 2 goal of their safety initiative, health care organizations began prioritizing alarm management programs, procedures, and policies. Unfortunately, according to a recent survey, few meaningful improvements in clinical alarm safety have been successfully implemented. ${ }^{2}$

Previous and current methods for monitoring real-time pulmonary function and for detecting respiratory depression include clinical observation, pulse oximetry, and capnography. Clinical observation is subjective and rarely continuous. Pulse oximetry and capnography are both plagued by nuisance alarms. The lack of reliable and actionable respiratory data has presented a unique challenge to anesthesiologists and perioperative professionals. ${ }^{4}$ While identifying and measuring hypoxia, pulse oximetry has been identified by nurses as the largest contributor of nuisance alarms..$^{5-7}$ The end result is that health care providers often lower alarm thresholds and reaction thresholds, increase latent time, or engage in a combination of both to avoid incessant alarms. ${ }^{4}$ Even worse, it has been reported that $78 \%$ of clinicians disable physiologic monitor alarms due to alarm fatigue, ${ }^{8}$ and $<1 \%$ overall alarms have resulted in changes of ongoing patient management. 9 The "cry wolf effect" has greatly hindered the effectiveness of respiratory monitoring systems despite their theoretical potential. It is critical that a clinically useful respiratory monitor be both reliable and provide actionable alarms.

Respiratory volume monitoring $(\mathrm{RVM})$ is a noninvasive technique that directly measures minute ventilation $\left(\dot{\mathrm{V}}_{\mathrm{E}}\right)$, tidal volume $\left(\mathrm{V}_{\mathrm{T}}\right)$, and breathing frequency. Most patients do not have a primary problem with oxygenation, but rather with insufficient ventilation. RVM provides continuous data and immediate feedback based on adequacy of ventilation, not just oxygenation. ${ }^{10}$ The RVM uses thoracic electrodes to collect electrical impedance data, present a

Correspondence: Chunyuan Qiu MD MSc, Department of Anesthesiology, Kaiser Permanente Baldwin Park Medical Center, 1011 Baldwin Park Blvd., Baldwin Park, CA 91706. E-mail: chunyuan.x.qiu@kp.org.

DOI: $10.4187 /$ respcare. 07187

\section{QUICK LOOK}

\section{Current knowledge}

Patients recovering from surgery are at risk of respiratory depression due to the combined effects of anesthesia and opioids, and monitoring is required to maintain patient safety. Current technologies for monitoring ventilation in patients recovering from surgery, such as pulse oximetry and capnography, are limited by indirect measurement of ventilation and are prone to false alarms.

\section{What this paper contributes to our knowledge}

We validated the use of the RVM to monitor minute ventilation in the perioperative setting. The RVM detected episodes of respiratory depression, resulting in actionable alarms and leading to interventions by medical staff. The RVM had a low false alarm rate, compared to pulse oximetry and capnography.

respiratory curve, and calculate $\dot{\mathrm{V}}_{\mathrm{E}}, \mathrm{V}_{\mathrm{T}}$, and breathing frequency values. The RVM has been shown to measure $\dot{V}_{\mathrm{E}}$ and $\mathrm{V}_{\mathrm{T}}$ with an accuracy error of approximately $10 \%$ and breathing frequency with an accuracy error of approximately $2 \%$ for adults and $12 \%$ and $4 \%$, respectively, for pediatric patients. ${ }^{10,11}$ Unlike peripheral capillary oxygen saturation $\left(\mathrm{S}_{\mathrm{pO}_{2}}\right)$ or partial pressure of end-tidal $\mathrm{CO}_{2}$ $\left(\mathrm{P}_{\mathrm{ETCO}_{2}}\right)$ measurements, which have an inherent delay in response to changes in ventilation, RVM directly measures changes in ventilation. As such, it has the potential to be a leading indicator of respiratory compromise or other change in respiratory status.

While previous research demonstrated that RVM can potentially lower the incidence of false alarms in the post-anesthesia care unit (PACU) compared to pulse oximetry, ${ }^{12}$ the actual alarm rate of RVM has not been evaluated. Therefore, we sought to evaluate the use of RVM in the perioperative setting at multiple centers to characterize the clinical use of the RVM during the perioperative period in the PACU, including alarm rates of RVM along with corresponding interventions by clinical staff. We hypothesized that a majority of RVM alarms would be actionable rather than nuisance alarms and thus would lead to intervention by medical staff. We also hypothesized that RVM would have fewer technical alarms than pulse oximetry or capnography monitoring systems. The study also explored whether reported results from other medical centers and from the Emergency Care Research Institute were reproducible within the Kaiser Permanente Medical System to assess the potential system-wide application of RVM. 


\section{Methods}

\section{Study Design}

Our quality improvement study was conducted at 4 hospital sites in the Kaiser Permanente system between July 17 and September 22, 2017, and included 247 subjects (California sites included Baldwin Park Medical Center, Fontana Medical Center, and San Jose Medical Center; the Oregon site was Sunnyside Medical Center, Clackamas, Oregon). The study was reviewed and approved by the Kaiser Permanente Institutional Review Board. No informed consent was required. Subjects who were 21$99 \mathrm{y}$ old in the perioperative period were included. Exclusion criteria were patients with allergies to adhesive electrodes and prior pneumonectomy or pulmonary lobectomy. Subjects considered to be at higher risk for respiratory complications were selected by hospital staff at each site based on patient history and demographics (ie, history of obstructive sleep apnea, obesity, elderly age, opioid sensitivity, or hemidiaphragmatic paralysis post nerve block). The complexity and type of surgery (orthopedics, general, bariatric, gynecological, or urological) and overall clinical impression were also considered.

\section{Equipment}

The ExSpiron 1Xi RVM (Respiratory Motion, Waltham, Massachusetts) was used to monitor respiratory status in all subjects in addition to standard clinical monitoring and according to each hospital's standard protocols. The ExSpiron-1Xi monitors and consumable ExSpiron PadSets were provided free of charge to the study sites by the manufacturer. Clinical staff were trained prior to the study on how to use the ExSpiron monitoring system. Data from the RVM along with other monitoring systems (when available) were used to assist with patient care. De-identified RVM data were extracted directly by staff on a daily basis for all subjects for the entire time they were enrolled in the study. Study data associated with study numbers were then paired with associated clinical data.

The RVM is a noninvasive impedance-based monitor that provides continuous real-time measurements of $\dot{V}_{E}$, $\mathrm{V}_{\mathrm{T}}$, and breathing frequency. ${ }^{10-15}$ The impedance signal was recorded via an electrode PadSet, which was placed on the patient with electrodes at the sternal notch, xiphoid, and the mid-axillary line at the level of the xiphoid. ${ }^{16}$ The RVM automatically calculated $\dot{\mathrm{V}}_{\mathrm{E}}, \mathrm{V}_{\mathrm{T}}$, and breathing frequency from the impedance signal with an average error of $\sim 10 \%$ for $\dot{\mathrm{V}}_{\mathrm{E}}$ and $\mathrm{V}_{\mathrm{T}}$, and $\sim 2 \%$ for breathing frequency relative to a spirometer. ${ }^{10}$ Subjects were monitored in the basic monitoring mode, which does not require any calibration with a spirometer or ventilator.
$\dot{\mathrm{V}}_{\mathrm{E}}$ is the primary output metric of the RVM and is used to trigger a low $\dot{\mathrm{V}}_{\mathrm{E}}$ alarm, corresponding to the eventual onset of respiratory insufficiency. The $\dot{V}_{\mathrm{E}}$ value displayed on the RVM represents a 30-s measurement of respiration that is updated every $5 \mathrm{~s}$ in a rolling fashion. To account for patients of various sizes and $\dot{V}_{\mathrm{E}}$ requirements, the RVM compares a patient's recorded $\dot{V}_{E}$ to a predicted $\dot{\mathrm{V}}_{\mathrm{E}}$ as a percentile. This is calculated using a body surface area formula based on the patient's height, weight, and gender. The threshold for a low $\dot{\mathrm{V}}_{\mathrm{E}}$ alarm was set to $40 \%$ of predicted $\dot{\mathrm{V}}_{\mathrm{E}}$ sustained for $2 \mathrm{~min}$ as described in the literature. ${ }^{12,13,17}$ Because previous studies have demonstrated that breathing frequency alone is a poor indicator of respiratory sufficiency, missing $>80 \%$ of low $\dot{V}_{E}$ measurements, ${ }^{18,19}$ low breathing frequency alarms were not enabled. Importantly, many patients with obstructive sleep apnea have prolonged apneas followed by large "rescue breaths" but maintain an adequate $\dot{\mathrm{V}}_{\mathrm{E}}$, so low breathing frequency alarms can lead to multiple nuisance alarms in patients with overall adequate ventilation.

With standard use, RVM reports 2 types of physiologic alarms: low $\dot{\mathrm{V}}_{\mathrm{E}}$ alarms were triggered by a decrease in $\dot{\mathrm{V}}_{\mathrm{E}}$ below $40 \%$ of predicted $\dot{\mathrm{V}}_{\mathrm{E}}$ sustained for $2 \mathrm{~min}$, and No Breath Detected alarms were triggered by an apnea lasting $>30 \mathrm{~s}$. Technical alarms reported by the RVM included signal interference, adhesive pad detachment, low battery, and nurse call button disconnections.

Subjects were monitored in both the PACU and on the general hospital floor. In the PACU, all subjects were continuously monitored with the RVM, and a subset continued to be monitored on the general hospital floor. A subset of subjects, based on their clinical needs and standard protocols, were placed on continuous monitoring with bedside pulse oximetry, bedside capnography, or telemetry pulse oximetry on the general hospital floor. Each monitoring technology had its own specific alarm conditions and corresponding alarm thresholds, which had been established as part of the care plan at each facility prior to this study and were not altered for the purposes of this study. For the purpose of comparison across technologies, alarms were categorized as either physiologic or technical (nuisance). Device descriptions and corresponding alarm conditions and threshold ranges are detailed below.

\section{Bedside Pulse Oximetry and Capnography Monitor}

The bedside monitor used in the study was the Philips SureSigns VM8 (Philips Medical Systems, Andover, Massachusetts), which continuously measures both $\mathrm{S}_{\mathrm{pO}_{2}}$ and $\mathrm{P}_{\mathrm{ETCO}_{2}}$. Physiologic alarms reported by the monitor were low $\mathrm{S}_{\mathrm{pO}_{2}}(<88 \%)$, low $\mathrm{P}_{\mathrm{ETCO}_{2}}(<10 \mathrm{~mm} \mathrm{Hg})$, high $\mathrm{P}_{\mathrm{ETCO}_{2}}$ ( $>60 \mathrm{~mm} \mathrm{Hg}$ ), low breathing frequency $(<6$ breaths $/ \mathrm{min})$, high breathing frequency ( $>35$ breaths $/ \mathrm{min}$ ), and apnea $(>30 \mathrm{~s})$. The following alarms reported by the monitor were 
considered technical: $\mathrm{S}_{\mathrm{pO}_{2}}$ sensor off, $\mathrm{S}_{\mathrm{pO}_{2}}$ no pulse, $\mathrm{S}_{\mathrm{pO}_{2}}$ poor signal, $\mathrm{S}_{\mathrm{pO}_{2}}$ interference, $\mathrm{CO}_{2}$ pump off, $\mathrm{CO}_{2}$ no tubing, and $\mathrm{CO}_{2}$ occlusion. Note that nasal cannula dislodgement often results in apnea or low breathing frequency alarms, which are false physiologic alarms. Unfortunately, in this study every alarm could not be deconvoluted into true or false, and are thus only categorized as physiologic or technical.

\section{Telemetry Pulse Oximetry Monitor}

The telemetry monitor $\left(\mathrm{S}_{\mathrm{pO}_{2}}\right.$ monitor, Philips) was supervised by telemetry technicians who categorized alarms as low $\mathrm{S}_{\mathrm{pO}_{2}}$ (ie, a physiologic alarm with $\mathrm{S}_{\mathrm{pO}_{2}}<90 \%$ ) or technical alarm corresponding to a poor quality $\mathrm{S}_{\mathrm{pO}_{2}}$ waveform resulting from artifact. Note that the alarms discussed in this study correspond to every alarm reported by the telemetry monitor and not just those relayed by the technicians to the care providers.

\section{Observations of Interventions}

The responses of clinical staff to RVM alarms were recorded and analyzed by nonclinical personnel. Alarms were divided into 4 categories: (1) actionable and addressed, (2) actionable and not addressed, (3) self-corrected, and (4) technical (nuisance). The action taken to resolve each alarm was recorded and further categorized. Self-corrected alarms were those that resolved without staff intervention, usually by the patient being stimulated by the audible RVM alarm. Technical alarms did not require intervention and were considered nuisance alarms.

The alarms for each of the monitoring devices were collected from the digital alarm log at the conclusion of monitoring. All alarms were audible at the bedside both in the PACU and on the general hospital floor. RVM alarms on the hospital floor were reported centrally through the nurse call system.

\section{Data Analysis}

$\dot{\mathrm{V}}_{\mathrm{E}}$ was expressed as a percent of each subject's predicted $\dot{V}_{\mathrm{E}}$, calculated based on the subject's body surface area and sex. ${ }^{13,15,20}$ A low $\dot{V}_{E}$ alarm was defined as a period of $\dot{\mathrm{V}}_{\mathrm{E}}<40 \%$ predicted lasting $\geq 2 \mathrm{~min}$. $\dot{\mathrm{V}}_{\mathrm{E}}<40 \%$ predicted was chosen as a parameter based on the Acute Respiratory Distress Syndrome Network (ARDSnet) protocol for successful extubation. ${ }^{13}$ No breath detected was defined as a respiratory pause of $\geq 30$. Technical alarms were defined as alarms which were neither low $\dot{\mathrm{V}}_{\mathrm{E}}$ nor no breath detected. Relative alarm rate was calculated as the number of alarm events divided by the length of monitoring.
Table 1. Anthropometric Data for All Subjects

\begin{tabular}{lcc}
\hline \hline & PACU & General Hospital Floor \\
\hline Subjects, $n$ & 247 & 109 \\
Male:Female ratio & $104: 143$ & $42: 67$ \\
Height, cm & $167.6 \pm 11.4$ & $166.5 \pm 11.5$ \\
Weight, $\mathrm{kg}$ & $91.1 \pm 27.1$ & $89.5 \pm 27.3$ \\
Body mass index, $\mathrm{kg} / \mathrm{m}^{2}$ & $32.3 \pm 8.7$ & $32.1 \pm 8.0$ \\
Age, $\mathrm{y}$ & $60.9 \pm 13.9$ & $64.1 \pm 11.1$ \\
& \\
Data are presented as $n$ subjects or mean $\pm \mathrm{SD}$. & \\
PACU = post-anesthesia care unit & \\
\hline
\end{tabular}

Mann-Whitney $U$ test was used to compare the relative alarm rates across monitoring technologies (RVM vs capnograph and/or pulse oximeter) for only the subset of subjects that had multiple monitoring technologies. Data are presented as mean $\pm \mathrm{SD}$ or median (interquartile range [IQR]), as specified in the text. All analyses were performed in Matlab 2014b (MathWorks, Natick, Massachusetts). Results were considered statistically significant at $P<.05$.

\section{Results}

\section{Demographics}

A total of 247 subjects were monitored for up to $48 \mathrm{~h}$ with the RVM at 4 hospital sites. Data collection was initiated in the PACU. All 247 subjects were monitored with the RVM and with standard bedside monitor parameters, including pulse oximetry and breathing frequency in the PACU, and 109 subjects had RVM continued on the general hospital floor after transfer. The choice of subjects for continuation of RVM monitoring was based on transfer to floors on which training on use of the RVM had been previously provided to the clinical staff.

Table 1 summarizes the demographics of all subjects studied and includes a breakdown of subjects who had RVM monitoring on the general hospital floor and/or PACU. The patient population spanned a wide range of age, height, weight, and body mass index values. The study targeted subjects considered by anesthesiology staff to be at risk of respiratory depression, such as those who were frail or had obstructive sleep apnea, obesity, COPD, congestive heart failure, opioid sensitivity, opioid tolerance, or hemidiaphragmatic paralysis post nerve block, or were undergoing major procedures (primarily general, gynecologic, orthopedic, and bariatric procedures). Most subjects received opioids, particularly those undergoing painful general, bariatric, and orthopedic surgeries. 
Table 2. Summary of Overall Monitored Time and RVM Alarms per Environment

\begin{tabular}{lccc}
\hline \hline & PACU & $\begin{array}{c}\text { General Hospital } \\
\text { Floor }\end{array}$ & Total \\
\hline Length of monitoring, h & 577 & 1,744 & 2,321 \\
Total alarms, no. (\%) & 235 & 370 & 605 \\
$\quad$ Low $\dot{V}_{\text {E }}$ alarms & $110(47)$ & $241(65)$ & $351(58)$ \\
$\quad$ Apnea alarms & $115(49)$ & $105(28)$ & $220(36)$ \\
$\quad$ Technical alarms & $10(4)$ & $24(7)$ & $34(6)$ \\
Alarm-free time, h & 425 & 1,524 & 1,949 \\
Percent of hours alarm- & 73.7 & 87.4 & 84 \\
$\quad$ free & & & 2.45 \\
Total alarms per patient & 0.95 & 3.39 & 0.26 \\
Total alarms per & 0.42 & 0.21 & \\
$\quad$ monitored hour & & & \\
Incidence of alarms, $n(\%)$ & & & \\
$\quad$ 0 Alarms & $153(62)$ & $44(40)$ & \\
1-3 Alarms & $80(32)$ & $30(28)$ & \\
$\quad$ Multiple alarms & $14(6)$ & $35(32)$ & \\
& & & \\
RVM = respiratory volume monitoring & & & \\
PACU = post-anesthesia care unit & &
\end{tabular}

\section{RVM Alarm Rates in PACU and on General Hospital Floor}

Subjects were monitored via RVM for a total of 2,321 h across the 4 hospitals. Of the total hours, $1,744 \mathrm{~h}$ were on the general hospital floor, and 1,524 of the 1,744 h (87\%) were free from alarms. More importantly, despite selection of higher-risk subjects, more than two thirds of all subjects on the hospital floor and $90 \%$ of the subjects in the PACU had either no alarms or very few (ie, 1-3) alarms (Table 2).

Figure 1 summarizes the relative alarm rate (ie, the number of alarms normalized by the total monitoring time for each subject) for technical, low $\dot{\mathrm{V}}_{\mathrm{E}}$, and no breath detected alarms in both the PACU and floor. Note that the RVM's technical alarms were not only rare $(0.02 / \mathrm{h}$ in the PACU and $0.01 / \mathrm{h}$ on the hospital floor), but they also represent $<5 \%$ of all alarms. This suggests that $>95 \%$ of RVM alarms were physiological and thus actionable. The relative alarm rate in the PACU was double the alarm rate on the hospital floor $(0.42 / \mathrm{h}$ vs $0.21 / \mathrm{h})$. The relative incidence of respiratory compromise (low $\dot{\mathrm{V}}_{\mathrm{E}}$ ) was similar between the PACU and the hospital floor $(0.20 / \mathrm{h}$ vs $0.16 / \mathrm{h}$ ). Interestingly, the relative incidence of long apneas (no breath detected for $30 \mathrm{~s}$ ) was 3 -fold higher in the PACU than on the hospital floor $(0.20 / \mathrm{h}$ vs $0.06 / \mathrm{h})$, which likely represents the effect of residual anesthesia.

\section{Interventions in Response to RVM Alarms}

Clinical staff received brief instructions regarding the use of the RVM, including that, if it alarmed, they should

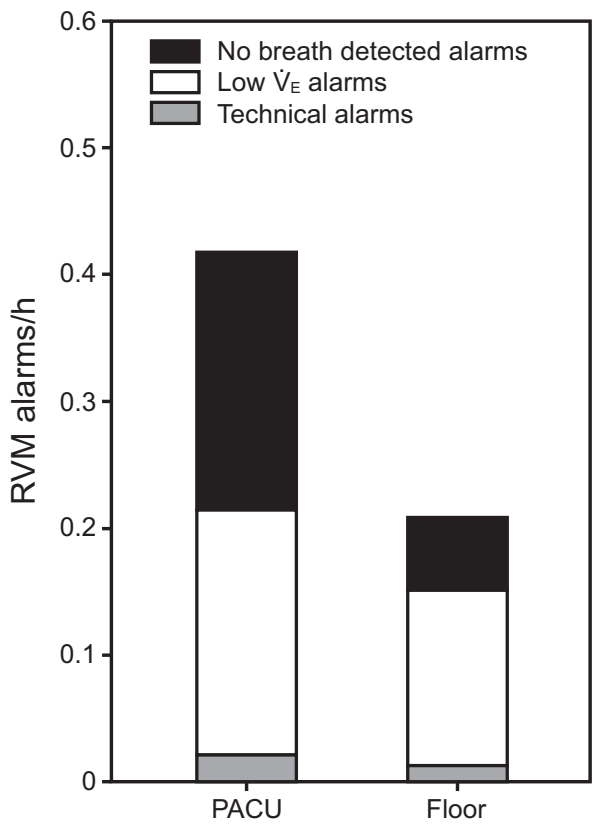

Fig. 1. Relative alarm rate per monitored hour for RVM in the PACU ( $n=247$ subjects) and on the general floor ( $n=109$ subjects). PACU = post-anesthesia care unit; RVM = respiratory volume monitoring; $\dot{\mathrm{V}}_{\mathrm{E}}=$ minute ventilation.

assess the subject's respiratory status and overall clinical picture. Nursing staff responded to and addressed most of the RVM alarms that were not self-resolved. Across all 4 clinical sites, $<6 \%$ of alarms were technical and considered nuisance alarms; $13 \%$ of all alarms were selfresolved, and $64 \%$ were resolved by an intervention, leaving $<20 \%$ of the alarms as "not addressed." This trend was similar across all 4 sites, as summarized in Figure 2 and Table 3. Note that the ratio of addressed to not-addressed alarms was slightly higher in the PACU (69\%: $15 \%)$ compared to the general hospital floor (61\%:18\%), likely due to higher staffing ratios in the PACU.

The most common intervention that was required to address an RVM physiological alarm was direct patient stimulation. This accounted for $65 \%$ of all interventions in the PACU and almost $79 \%$ of all interventions on the general hospital floor (Table 4). Additional interventions included repositioning the subject, a change in opioid dosing, and initiation of CPAP or bi-level positive airway pressure. About $2 \%$ of subjects required further intervention from a physician, respiratory therapy, or an increase in level of care. Note that the number of listed interventions in Table 4 exceeds the number of actionable alarms that were addressed because more than one intervention or action could have been initiated in response to a single alarm.

In addition to addressing physiological alarms, the RVM was also used to alter care in subjects without any alarms. The actions staff took based on the RVM data in subjects without any alarms were primarily related to assurance of 


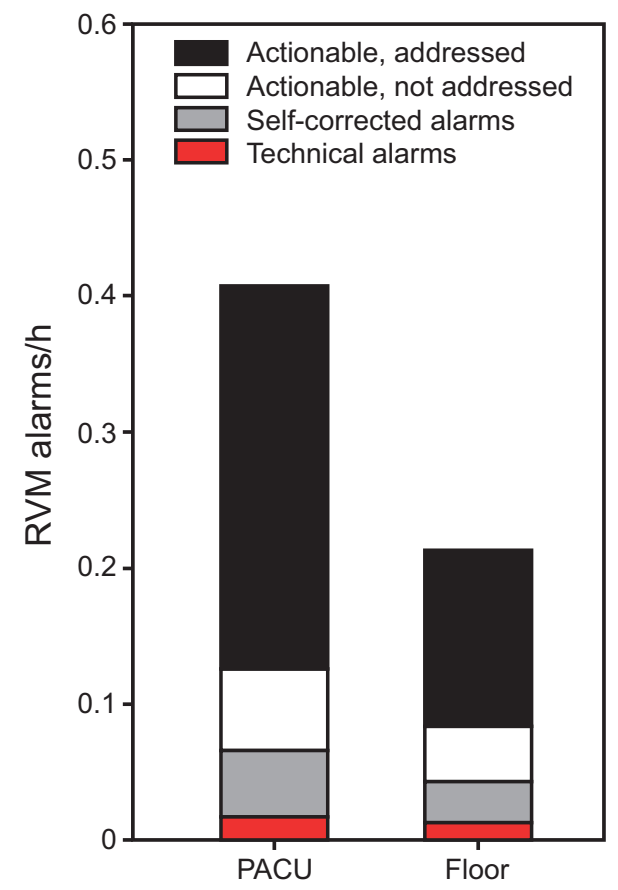

Fig. 2. Comparison on the RVM alarms that were addressed, not addressed, self-corrected, and technical alarms across all clinical sites in the PACU and on the floor. PACU = post-anesthesia care unit; RVM = respiratory volume monitoring.

Table 3. Summary of RVM Alarm Categories

\begin{tabular}{lccc}
\hline \hline & Total & PACU & $\begin{array}{c}\text { General Hospital } \\
\text { Floor }\end{array}$ \\
\hline Total alarms & 605 & 235 & 370 \\
Technical alarms & $34(6)$ & $10(4)$ & $24(7)$ \\
Self-corrected alarms & $79(13)$ & $28(12)$ & $51(14)$ \\
Actionable alarms, & $102(17)$ & $34(14)$ & $68(18)$ \\
$\quad$ not addressed & & & \\
Actionable alarms, addressed & $390(64)$ & $163(69)$ & $227(61)$ \\
& & & \\
$\begin{array}{l}\text { Data are presented as no. (\%). } \\
\text { RVM }=\text { respiratory volume monitoring }\end{array}$ & & & \\
PACU = post-anesthesia care unit & & & \\
& & & \\
\hline
\end{tabular}

adequate ventilation. These included an increase or decrease in opioid dosing, avoiding the need for intubation, early discharge, or transfer to a lower acuity setting. Table 5 details the number of subjects who had actions or interventions associated with alarms. For subjects with alarms, a small number of subjects experienced alarms that were not addressed (17\% in the PACU and $15 \%$ on the hospital floor), whereas most subjects had all of their alarms addressed by actions or interventions (69\% in the PACU and $57 \%$ on the hospital floor). For subjects with no alarms, the majority (92\% in the PACU and $98 \%$ on the hospital floor) had no actions taken.
Table 4. Detailed Summary of Clinical Interventions in Response to Actionable (Physiological) RVM Alarms

\begin{tabular}{lccc}
\hline \hline & Total & PACU & $\begin{array}{c}\text { General Hospital } \\
\text { Floor }\end{array}$ \\
\hline Total interventions, no. & 490 & 239 & 251 \\
Stimulation & $355(72)$ & $156(65)$ & $199(79)$ \\
Increased opioids & $9(2)$ & $8(3)$ & $1(0.4)$ \\
Decreased opioids & $8(2)$ & $2(1)$ & $6(2)$ \\
Change in other medications & $9(2)$ & $7(3)$ & $2(1)$ \\
CPAP/BPAP & $16(3)$ & $4(2)$ & $12(5)$ \\
Subject repositioned & $15(3)$ & $5(2)$ & $10(4)$ \\
Physician/RT alerted & $8(2)$ & $3(1)$ & $5(2)$ \\
Change in level of care & $5(1)$ & $3(1)$ & $2(1)$ \\
Change in length of stay & $11(2)$ & $11(5)$ & $0(0)$ \\
Avoided intubation & $5(1)$ & $4(2)$ & $1(0.4)$ \\
& & & \\
Data are presented as no. (\%). & & & \\
RVM $=$ respiratory volume monitoring & & & \\
PACU = post-anesthesia care unit & & & \\
RT $=$ respiratory therapist & & & \\
\hline
\end{tabular}

\section{Comparison of Alarm Rates for RVM, Pulse Oximetry, and Capnography}

A subset of subjects $(n=7)$ were prescribed continuous pulse oximetry and capnography bedside monitoring on the general hospital floor. The relative incidence of alarms from the RVM and the bedside oximetry/capnography monitor was compared for only those subjects. For each of the 7 subjects, the number of alarms was normalized by the length of monitoring by each respective modality and then averaged across patients (Fig. 3A). The incidence of technical alarms for the bedside oximetry/capnography monitor $(9.4 \mathrm{alarms} / \mathrm{h})$, was almost 1,000-fold-higher compared to the RVM alarms ( 0.01 alarms/h). Importantly, the bedside capnography monitor cannot distinguish between an apnea in the capnography trace (as indicated by lack of change in the partial pressure of $\mathrm{CO}_{2}$ ) and a displaced sampling nasal cannula. Comparing the alarm rates per subject showed that capnography had a significantly higher rate of both physiological alarms (median [IQR]: 1.49 [0.97-5.56] vs $0.04[0-0.33]$ alarms/h, $P<.001)$ and technical alarms (median [IQR]: $5.80[1.89-10.25]$ vs $0[0-0]$ alarms $/ \mathrm{h}, P<.001)$ per hour per subject compared to the RVM.

Another subset of subjects $(n=7)$ at the same clinical site were prescribed only continuous pulse oximetry monitoring on the general hospital floor. Again we compared the relative incidence of alarms across monitoring modalities for this small subset of subjects, and not the entire population receiving RVM monitoring. Figure 3B summarizes these results, showing 0.24 physiologic alarms $/ \mathrm{h}$ on the RVM versus 0.5 physiologic alarms/h on the bedside pulse oximetry monitor. The disparity between technical alarms is again highly significant; nearly 50-fold, with 
Table 5. Subjects With and Without Actions or Interventions Based on RVM Data Across All Clinical Sites

\begin{tabular}{|c|c|c|c|c|c|}
\hline & \multicolumn{3}{|c|}{ Subjects With No Alarms } & \multicolumn{2}{|c|}{ Subjects With Alarms } \\
\hline & Action Taken & No Action Taken & Alarms Not Addressed & Some Alarms Addressed & All Alarms Addressed \\
\hline PACU & $13(8)$ & $140(92)$ & $16(17)$ & $13(14)$ & $65(69)$ \\
\hline General hospital floor & $1(2)$ & $43(98)$ & $10(15)$ & $18(28)$ & $37(57)$ \\
\hline \multicolumn{6}{|c|}{$\begin{array}{l}\text { Data are presented as } n(\%) . \\
\text { RVM }=\text { respiratory volume monitoring } \\
\text { PACU = post-anesthesia care unit }\end{array}$} \\
\hline
\end{tabular}
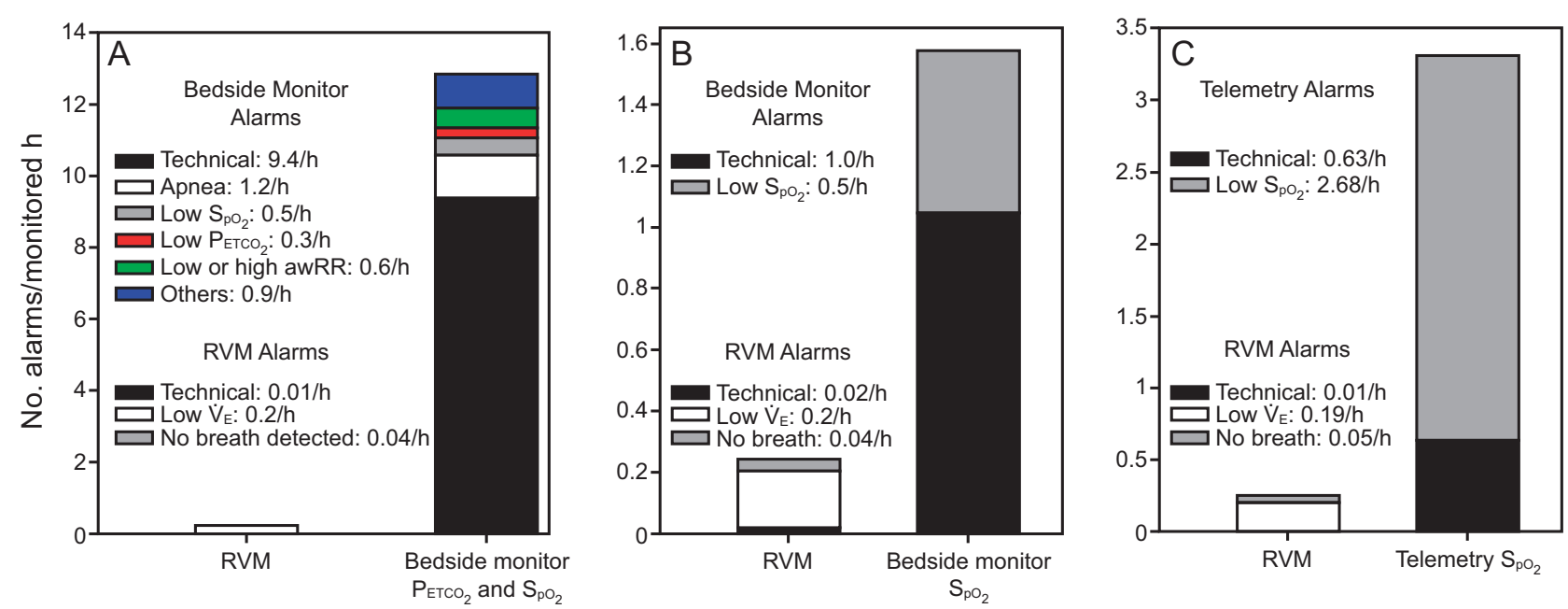

Fig. 3. The alarm rate per monitored hour for RVM, pulse oximetry, and capnography. (A) Simultaneous bedside $\mathrm{P}_{\mathrm{ETCO}_{2}}$ and $\mathrm{S}_{\mathrm{pO}} \mathrm{monitoring}_{2}$

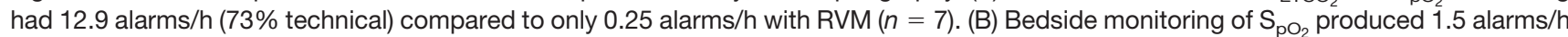
(67\% technical) compared to only 0.26 alarms/h with RVM $(n=7)$. (C) $\mathrm{S}_{\mathrm{pO}_{2}}$ monitoring by telemetry technicians reported 3.31 alarms/h ( $19 \%$ technical) compared to only 0.26 alarms/h with RVM $(n=15)$. awRR $=$ breathing frequency measured by capnography, RVM $=$ respiratory volume monitoring.

0.02 technical alarms/h on the RVM versus 1 technical alarm/h on the bedside oximetry monitor.

The third subset of subjects $(n=15)$ were prescribed telemetry pulse oximetry monitoring on the general hospital floor. Their data were used to cross-validate the results obtained from the bedside oximetry monitor (Fig. $3 \mathrm{C})$. We found that the incidence of pulse oximeter technical alarms was similar in the bedside and telemetry implementations. (1.0 vs 0.63 alarms/h) and still significantly (30-fold) higher that the RVM technical alarms (0.02 alarms/h). Table 6 summarizes the anthropometrics of all 3 subgroups whose data are presented in Figure 3.

Comparing RVM and pulse oximetry alarm rates for the 29 subjects monitored with both modalities across 2 sites, it is evident that these subjects had a significantly higher rate of both physiological alarms (median [IQR]: 0.67 [0.07-2.58] vs $0.24[0.03-0.50]$ alarms $/ \mathrm{h}, P=.02$ ) and technical alarms (median [IQR]: 0.47 [0.09-1.13] vs $0[0-0]$ alarms $/ \mathrm{h}, P<.001)$ per monitored hour generated by pulse oximetry than by RVM.
Table 6. Anthropometrics for the 3 Subsets of Subjects Monitored With RVM and Bedside or Telemetry Monitoring

\begin{tabular}{lccc}
\hline \hline & $\begin{array}{c}\text { Bedside } \mathrm{S}_{\mathrm{PO}_{2}} \\
\text { and } \mathrm{P}_{\mathrm{ETCO}_{2}}\end{array}$ & $\begin{array}{c}\text { Bedside } \\
\mathrm{S}_{\mathrm{pO}_{2}}\end{array}$ & $\begin{array}{c}\text { Telemetry } \\
\mathrm{S}_{\mathrm{pO}_{2}}\end{array}$ \\
\hline Subjects & 7 & 7 & 15 \\
Male:Female ratio & $3: 4$ & $3: 4$ & $4: 11$ \\
Height, cm & $166.4 \pm 16.0$ & $166.4 \pm 14.1$ & $165.8 \pm 9.4$ \\
Weight, $\mathrm{kg}$ & $77.1 \pm 12.9$ & $86.3 \pm 27.3$ & $110.6 \pm 29.7$ \\
Body mass index, $\mathrm{kg} / \mathrm{m}^{2}$ & $27.8 \pm 5.1$ & $30.7 \pm 7.2$ & $40.0 \pm 9.3$ \\
Age, y & $70.8 \pm 3.7$ & $62.8 \pm 12.3$ & $58.9 \pm 10.6$
\end{tabular}

$\overline{\text { Data are presented as }} n$ subjects or mean $\pm \mathrm{SD}$.

$\mathrm{RVM}=$ respiratory volume monitoring

\section{Discussion}

Routine vital signs, such as pulse oximetry, temperature, breathing frequency, and blood pressure, are clinical measurements that indicate the body's basic functions and 
disease status. More sophisticated physiological monitoring, especially for the detection of cardiovascular health, may include electrocardiography, invasive blood pressure, central venous pressure, cardiac output, echocardiography, etc. However, continuous noninvasive bedside measurement of pulmonary function has received less attention, and previous technologies have fallen short. An ideal device would be noninvasive, continuous, and provide accurate real-time readings of respiratory function and its compromise. Additionally, the device would have to be simple to apply, use, read, and interpret with few nuisance alarms.

Recent studies and guidelines have stressed the importance of accurate real-time respiratory monitoring to optimize patient safety. The newly available RVM monitor provides a welcome addition to the existing lineup of respiratory status monitoring. Unfortunately, the frequency of nuisance alarms presented from pulse oximeters and capnography monitors have predisposed hospital staff with a sense of caution in the introduction of new technology into routine clinical practice. This is primarily due to the fear of adding to existing alarm fatigue and contributing to additional burden to staff. Here we investigated the utility of RVM in the perioperative setting, with a focus on identifying actionable versus nuisance alarms. We then compared RVM alarm rates with alarm rates from pulse oximeters and capnography monitors in the same setting of the PACU and general hospital floors. Our results indicate that RVM alarms had far less nuisance alarms and fewer but more actionable alarms compared to capnography and/or pulse oximetry; RVM did not miss any episodes of subject deterioration; the RVM device was easy to use; and most issues raised by RVM alarms were early in the spiral of respiratory deterioration and thus easily addressed by nursing staff with minimal training, minimizing cost to the system.

The overall RVM alarm rate was significantly lower than with either pulse oximeters or capnography monitors. We saw a nearly 1,000 -fold reduction in nuisance alarms compared to capnography and a 20-50-fold reduction in nuisance alarms compared to pulse oximetry. These results were consistent and reproducible across all 4 clinical sites. Additionally, the majority of RVM alarms were actionable (as seen in Table 5). This included verbal or physical stimulation, narcotic dose adjustment, patient repositioning, CPAP application, or upgrade to a higher level of care. Subjects who may not have otherwise be given opioids due to their obstructive sleep apnea status or other concerns were able to be provided with increased analgesia and effective pain management based on the adequate ventilatory status demonstrated by the RVM. In other subjects, a modification of the clinical plan was implemented based on their depressed ventilatory status. Some subjects only required stimulation or a change in position to improve their ventilatory status. These were simple interven- tions to potentially prevent life-threatening outcomes. These simple interventions, based on early RVM data, are far preferable to what is often required when responding to the delayed responses of other monitoring systems that can put patients at greater risk and require more difficult and costly interventions.

We noted that RVM reliably alerted the clinical staff in both the PACU and general hospital floor to a clinically important reduction in ventilation. These changes were seen well before changes to $\mathrm{S}_{\mathrm{pO}_{2}}$. Changes in $\mathrm{P}_{\mathrm{ETCO}_{2}}$ were not reliably actionable or indicative of the subject status. These results support earlier reports from a group at Massachusetts General Hospital, which demonstrated that low $\dot{\mathrm{V}}_{\mathrm{E}}$ preceded significant desaturation with an average window of 71 min. ${ }^{12}$ The important issue of nuisance alarms was also addressed. Pulse oximeter and capnography alarms often fall into 2 categories: technical alarms that are not actionable and alarms that are urgent or emergent in nature. In contrast, the RVM provides an early warning capability before impending detrimental events, providing clinical staff with valuable time to intervene early and coordinate preemptive interventions.

We observed that the relative RVM alarm rate in the PACU was double the alarm rate reported on the general hospital floor. These findings were slightly different from those reported by Saasouh et $\mathrm{al}^{21}$ and Schumann et al, ${ }^{22}$ in which the incidence of respiratory depression was found to be greater between $3 \mathrm{~h}$ and $14 \mathrm{~h}$ postoperatively on the hospital floor than it was in the PACU. ${ }^{21,22}$ The disparity may reflect differences in study design and clinical practice as well as somewhat different settings. The end point in our study was the evaluation of physiologic versus nuisance alarms, whereas the end point in studies by Saasouh et $\mathrm{al}^{21}$ and Schumann et $\mathrm{al}^{22}$ was alarms due to respiratory compromise defined by low $\dot{\mathrm{V}}_{\mathrm{E}}$ but not apnea. It was also likely that the patient population or the nature of the anesthesia and analgesia protocols varied. Our study indicates that alarms due to long apneas ( $>30$ s) were 3 times more prevalent in the PACU than on the hospital floor, implying a residual effect of narcotics and anesthetics in the PACU. The differences and similarities suggest that the postoperative course of respiratory function recovery is a dynamic process with multiple factors. As such, return to baseline for respiratory function such as rate, rhythm, and volume all have highly variable rates, especially in the presence of obstructive sleep apnea. ${ }^{15}$ Our results highlight the importance and unmet need of a complete respiratory function monitor for the entire perioperative period.

All respiratory monitoring technologies have their own limitations. For example, pulse oximetry is critical in a situation of ongoing respiratory failure; however, it has limited use in detecting the early signs of impending respiratory depression. It is also known that the $\mathrm{S}_{\mathrm{pO}_{2}}$ value is less reliable when it begins to drop due to a larger error at 
lower readings. Unlike RVM, pulse oximetry monitoring also produces artifacts and alarms in situations of movement, temperature extremes, or due to peripheral vasoconstriction. $\mathrm{P}_{\mathrm{ETCO}_{2}}$ can be useful in detecting apnea, although it is inaccurate in reflecting the value of partial pressure of arterial $\mathrm{CO}_{2}$ in a variety of settings, including mouthbreathing patients and during use of open oxygen systems. ${ }^{23,24} \mathrm{P}_{\mathrm{ETCO}_{2}}$ also has limited use in detecting causes of respiratory compromise and can confuse respiratory and cardiac compromise.

RVM, as demonstrated here, is very useful as an early warning system for pending respiratory depression but does not specifically measure the gas exchange status. Due to the known effects of anesthesia, analgesia, and surgery, both individually and synergistically, precise respiratory function monitoring is critical in all aspects of care, especially during the perioperative period setting. While RVM can be used to safeguard vulnerable patients in the perioperative period, it may also be used to guide precise perioperative care, anesthesia, and analgesia to promote functional recovery. It can provide critical information both in the PACU as well as on the general hospital floor, in either post-surgical or nonsurgical patients who are at high risk. While these uses have been less well studied, there is clearly an unmet clinical need. With few nuisance alarms impacting workflow and early detection to enable a paced response instead of an emergent action, RVM may provide a useful tool for both the PACU and the general hospital floor. Additionally, the data presented here lay the groundwork for establishing care protocols based on RVM alarms. Nursing protocols outlining steps to be taken in response to RVM alarms can be constructed to standardize care and improve safety. One such protocol has been suggested: assess the patient clinically (including vital signs and oxygen saturation measurement) and consider one of: (1) stimulate; (2) reposition; (3) incentive spirometry; (4) cut opioids in half; (5) call physician. Because of the early nature of the RVM alarm, many of these interventions are free or inexpensive. Early in the spiral of respiratory failure, patients can be returned more quickly to homeostasis, enhancing safety and reducing additional costs to the health care system.

Interestingly, one of the most common clinical mistakes in monitoring and diagnosing pending respiratory compromise is the use and misuse of supplemental oxygen. As a therapeutic agent, like any other medication, supplemental oxygen has many proven clinical benefits and even newly recognized potential benefits, such as its application in reducing surgical site infection. ${ }^{25}$ However, the routine delivery of inspired oxygen at levels greater than atmospheric oxygen is not without consequences. ${ }^{26}$ The common practice of addressing and attempting to reverse arterial hypoxemia without a clear understanding of the underlying cause can be dangerous. This is especially true in the perioperative setting, where arterial hypoxemia is generally caused by hypoventilation. Profound hypoventilation can be masked by supplemental oxygen until the point of arterial desaturation; a phenomenon called oxygenation without ventilation. ${ }^{27}$ Conversely, supplemental oxygen without hypoventilation can produce a state of hyperoxia, which, in select patient groups, can disturb vital organ blood perfusion and increase mortality. ${ }^{26,28}$ Therefore, it is potentially beneficial to combine the use of RVM with pulse oximetry. Measurements of both ventilation and oxygenation can be used in tandem to make decisions regarding delivery of supplemental oxygen and ventilatory management. Continuous use of RVM with intermittent $\mathrm{S}_{\mathrm{pO}_{2}}$ measurement on a standard scheduled basis and during the evaluation initiated by an RVM alarm has been suggested. ${ }^{12}$

This study has several potential limitations. First, although nursing staff received training on RVM devices and suggestions as to possible interventions, there were no formal protocols or procedures implemented to direct actions associated with alarms or during the detection of hypoventilation. As a result, we cannot determine specific impacts of the monitor on clinical outcomes. In fact, as with all assessments of monitoring systems, the effect of RVM monitoring on the relevant clinical outcome is difficult to determine because outcomes are primarily based on therapeutic intervention. Therefore, a specific protocol assessing diagnostic/therapeutic intervention is required for proper assessment. Subsequently, there is little relevant outcomes data for even the commonly used oximetry and capnography monitors, and the impact of these devices on mortality and morbidity from anesthesia are yet to be confirmed ${ }^{24,29}$ Second, we did not assess the direct or indirect cost burden or overall cost savings that would be associated from the clinical implementation of the device. Third, we did not assess patient care experience in comparison with existing technology. Fourth, because this is a retrospective analysis, a properly design prospective randomized study would need to be designed to determine the effectiveness of the RVM in a clinical setting. Finally, the sample sizes were relatively low and the settings were also limited to a single health care system. As such, the results may be biased despite the clinical experience and effective implementation that was experienced by staff.

\section{Conclusions}

Clinical advances in new monitoring products and solutions continue to be at the leading edge of surgical and perioperative research. One of the greatest limiting factors, in terms of implementation, is the redundancy and frequency of nuisance alarms, which leads to an increased rate of ignoring or silencing alarms. The optimum monitoring system must 
provide reliable real-time values that are actionable (ie, from which a clinical action can be determined) and provide early detection and warning of pending deterioration, so that early, small interventions can prevent the need for later, larger interventions. It must provide this early information without nuisance alarms that distract from patient care. This multicenter study demonstrated that the RVM system provided both an increase in actionable alarms as well as a decrease in nuisance alarms compared to pulse oximetry and capnography monitoring systems. In both PACU and general hospital floor perioperative settings, the RVM provided reliable, effective, and accurate measurements of respiratory status. Based on these findings, the RVM has the potential to provide major safety and economic benefits for patients, caregivers, and hospital systems alike.

\section{REFERENCES}

1. Shetty RM, Bellini A, Wijayatilake DS, Hamilton MA, Jain R, Karanth S, Namachivayam A. BIS monitoring versus clinical assessment for sedation in mechanically ventilated adults in the intensive care unit and its impact on clinical outcomes and resource utilization. Cochrane Database Syst Rev 2018;2:CD011240.

2. Ruppel H, Funk M, Clark JT, Gieras I, David Y, Bauld TJ, et al. Attitudes and practices related to clinical alarms: a follow-up survey. Am J Crit Care 2018;27(2):114-123.

3. The Joint Commission. National Patient Safety Goals. Available at: https://www.jointcommission.org/assets/1/6/2015_NPSG_HAP. pdf. Accessed August 7, 2019.

4. Tsien CL, Fackler JC. Poor prognosis for existing monitors in the intensive care unit. Crit Care Med 1997;25(4):614-619.

5. Downs JB. Prevention of hypoxemia: the simple, logical, but incorrect solution. J Clin Anesth 1994;6(3):180-181.

6. Downs JB. Has oxygen administration delayed appropriate respiratory care? Fallacies regarding oxygen therapy. Respir Care 2003; 48(6):611-620.

7. Jabre P, Jacob L, Auger H, Jaulin C, Monribot M, Aurore A, et al. Capnography monitoring in nonintubated patients with respiratory distress. Am J Emerg Med 2009;27(9):1056-1059.

8. Atzema C, Schull MJ, Borgundvaag B, Slaughter GR, Lee CK. ALARMED: adverse events in low-risk patients with chest pain receiving continuous electrocardiographic monitoring in the emergency department. A pilot study. Am J Emerg Med 2006;24(1): $62-67$.

9. Welch J. An evidence-based approach to reduce nuisance alarms and alarm fatigue. Biomed Instrum Technol 2011;Spring(Suppl):46-52.

10. Voscopoulos C, Brayanov J, Ladd D, Lalli M, Panasyuk A, Freeman J. Evaluation of a novel noninvasive respiration monitor providing continuous measurement of minute ventilation in ambulatory subjects in a variety of clinical scenarios. Anesth Analg 2013;117(1):91-100.

11. Gomez-Morad AD, Cravero JP, Harvey BC, Bernier R, Halpin E, Walsh B, Nasr VG. The evaluation of a noninvasive respiratory volume monitor in pediatric patients undergoing general anesthesia. Anesth Analg 2017;125(6):1913-1919.

12. Galvagno SM Jr, Duke PG, Eversole DS, George EE. Evaluation of respiratory volume monitoring (RVM) to detect respiratory compromise in advance of pulse oximetry and help minimize false desaturation alarms. J Trauma Acute Care Surg 2016;81(5 Suppl 2):S162-S170.

13. Voscopoulos CJ, MacNabb CM, Freeman J, Galvagno SM Jr, Ladd D, George E. Continuous noninvasive respiratory volume monitoring for the identification of patients at risk for opioid-induced respi- ratory depression and obstructive breathing patterns. J Trauma Acute Care Surg 2014;77(3 Suppl 2):S208-S215.

14. Mehta JH, Williams GW 2nd, Harvey BC, Grewal NK, George EE. The relationship between minute ventilation and end tidal $\mathrm{CO} 2$ in intubated and spontaneously breathing patients undergoing procedural sedation. PLoS One 2017;12(6):e0180187.

15. Schumann R, Kwater AP, Bonney I, Ladd D, Kim J, Gupta A, et al. Respiratory volume monitoring in an obese surgical population and the prediction of postoperative respiratory depression by the STOPbang OSA risk score. J Clin Anesth 2016;34:295-301.

16. Voscopoulos CJ, MacNabb CM, Brayanov J, Qin L, Freeman J, Mullen GJ, et al. The evaluation of a non-invasive respiratory volume monitor in surgical patients undergoing elective surgery with general anesthesia. J Clin Monit Comput 2015;29(2):223-230.

17. Voscopoulos C, Theos K, Tillmann Hein HA, George E. A risk stratification algorithm using non-invasive respiratory volume monitoring to improve safety when using post-operative opioids in the PACU. J Clin Monit Comput 2017;31(2):417-426.

18. Holley K, MacNabb CM, Georgiadis P, Minasyan H, Shukla A, Mathews D. Monitoring minute ventilation versus respiratory rate to measure the adequacy of ventilation in patients undergoing upper endoscopic procedures. J Clin Monit Comput 2016;30(1):33-39.

19. Cavalcante AN, Martin YN, Sprung J, Imsirovic J, Weingarten TN. Low minute ventilation episodes during anesthesia recovery following intraperitoneal surgery as detected by a non-invasive respiratory volume monitor. J Clin Monit Comput 2018;32(5):929-935.

20. Du Bois D, Du Bois EF. A formula to estimate the approximate surface area if height and weight be known. 1916. Nutrition 1989; 5(5):303-311.

21. Saasouh W, Hovsepyan K, Harvey B, Chang C, Turan A. Respiratory status at PACU discharge as predictor for post-operative respiratory depression on the general hospital floor (abstract). Anesth Analg 2017;124(Suppl 2):850-852.

22. Schumann R, Harvey B, Zahedi F, Bonney I. Minute ventilation assessment in the PACU is useful to predict postoperative respiratory depression following discharge to the floor: a prospective cohort study. J Clin Anesth 2019;52:93-98.

23. Takano Y, Sakamoto O, Kiyofuji C, Ito K. A comparison of the endtidal $\mathrm{CO} 2$ measured by portable capnometer and the arterial $\mathrm{PCO} 2$ in spontaneously breathing patients. Respir Med 2003;97(5):476-481.

24. Lam T, Nagappa M, Wong J, Singh M, Wong D, Chung F. Continuous pulse oximetry and capnography monitoring for postoperative respiratory depression and adverse events: a systematic review and meta-analysis. Anesth Analg 2017;125(6):2019-2029.

25. Belda FJ, Aguilera L, García de la Asunción J, Alberti J, Vicente R, Ferrándiz L, et al. Supplemental perioperative oxygen and the risk of surgical wound infection: a randomized controlled trial. JAMA 2005; 294(16):2035-2042.

26. Girardis M, Busani S, Damiani E, Donati A, Rinaldi L, Marudi A, et al. Effect of conservative vs conventional oxygen therapy on mortality among patients in an intensive care unit: the oxygen-ICU randomized clinical trial. JAMA 2016;316(15):1583-1589.

27. van Loon K, van Rheineck Leyssius AT, van Zaane B, Denteneer M, Kalkman CJ. Capnography during deep sedation with propofol by nonanesthesiologists: a randomized controlled trial. Anesth Analg 2014;119(1):49-55.

28. Durlinger EMJ, Spoelstra-de Man AME, Smit B, de Grooth HJ, Girbes ARJ, Oudemans-van Straaten HM, Smulders YM. Hyperoxia: At what level of $\mathrm{SpO} 2$ is a patient safe? A study in mechanically ventilated ICU patients J Crit Care 2017;39:199-204.

29. Pedersen T, Nicholson A, Hovhannisyan K, Møller AM, Smith AF, Lewis SR. Pulse oximetry for perioperative monitoring. Cochrane Database Syst Rev 2014;3:CD002013. 